\title{
PATTERNS IN A BALANCED BISTABLE EQUATION WITH HETEROGENEOUS ENVIRONMENTS ON SURFACES OF REVOLUTION
}

\author{
MAICON SÔNEGO
}

Abstract. We use the variational concept of $\Gamma$-convergence to obtain sufficient conditions that guarantee existence, stability and the geometric structure of four families of stationary solutions to the singularly perturbed parabolic equation $\partial_{t} u_{\varepsilon}=\varepsilon^{2} \Delta u_{\varepsilon}+f\left(u_{\varepsilon}, x\right)$ on surfaces of revolution. We consider the bistable function $f(u, x)=-(u-a(x))(u-b(x))(u-c(x))$ and the conditions found relate the functions $a, b, c$ to the geometry of the surface where such functions are defined.

Mathematics subject classification (2010): 35K57, 35B36, 35R01, 35B25, 35B35, 34K20, $58 \mathrm{~J} 32$. Keywords and phrases: patterns, $\Gamma$-convergence, surface of revolution, stable transition layer.

\section{REFERENCES}

[1] C. BAndle, F. Punzo And A. TESEI, Existence and nonexistence of patterns on Riemannian manifolds, J. Math. Anal. Appl. 387 (2012), 33-47.

[2] A. L. BESSE, Manifolds all of whose Geodesics are Closed, Springer, 1978.

[3] R. G. CASTEN AND C. J. Holland, Instability results for reaction diffusion equations with Neumann boundary conditions, J. Diff. Eqns., 2 (1978), 266-273.

[4] A. S. Do NAscimento, Stable stationary solutions induced by spatial inhomogeneity via $\Gamma$ convergence, Bulletin of the Brazilian Mathematical Society, 29 No.1 (1998), 75-97.

[5] A. S. Do NASCIMENTO, On the role of diffusivity in some stable equilibria of a diffusion equation, J. Diff. Eqns., 155 No. 2 (1999), 231-244.

[6] A. S. Do NASCIMENTO AND M. Sônego, Patterns on surfaces of revolution in a diffusion problem with variable diffusivity, Electr. J. Diff. Eqns., 2014, (2014) No. 238, 1-13.

[7] A. S. Do NAScimento AND M. Sônego, The roles of diffusivity and curvature in patterns on surfaces of revolution, J. of Math. Anal. and Appl. Vol. 412 (2014), 1084-1096.

[8] A. S. Do NAScimento And M. Sônego, Stable equilibria to a singularly perturbed reactiondiffusion equation in a degenerated heterogeneous environment, J. of Math. Anal. and Appl. Vol. 433 (2016), 1743-1756.

[9] A. S. Do NAscimento And M. Sônego, Stable Transition Layers to Singularly Perturbed Spatially Inhomogeneous Allen-Cahn Equation, Advanced Nonlinear Studies 15 (2015), 363-376.

[10] E. Giusti, Minimal Surfaces and Functions of Bounded Variation, Birkhauser, 1984.

[11] D. Henry, Geometric Theory of Semilinear Parabolic Equations, Springer Lecture Notes in Mathematics, 840, 1981.

[12] R. V. Kohn AND P. Sternberg, Local minimizers and singular pertubations, Proceedings of the R. Soc. of Edinburg, 111 (1989), 69-84.

[13] K. Kurata, H. Matsuzawa, Multiple stable patterns in a balanced bistable equation with heterogeneous environments, Applicable Analysis, 89 No. 7 (2010), 1023-1035.

[14] F. Li, K. NAKAshima, Transition Layers for a spatially inhomogeneous Allen-Cahn equation in multi-dimensional domains, Discrete Contin. Dyn. Syst., 32 No. 4 (2012), 1391-1420.

[15] F. Mahmoudi, A. Malchiodi And J. WeI, Transition layer for the heterogeneous Allen-Cahn equation, Ann. I. H. Poincare A.N., 25 (2008), 609-631.

[16] H. Matano, Asymptotic behavior and stability of solutions of semilinear diffusion equations, Publ. RIMS, Kyoto Univ., 15 (1979), 401-454. 
[17] H. MatsuZawa, Stable transition layers in a balanced bistable equation with degeneracy, Nonlinear Analysis, 58 (2004), 45-67.

[18] K. NAKASHIMA, Stable transition layers in a balanced bistable equation, Differential Integral Equations, 13 No. 7-9 (2000), 1025-1038.

[19] F. Punzo, The existence of patterns on surfaces of revolution without boundary, Nonlinear Analysis, 77 (2012), 94-102.

[20] J. SMOLLER, Shock Waves and Reaction - Diffusion Equations, Springer New York, 258, 1994.

[21] P. Sternberg, The effect of a singular pertubation on nonconvex variational problems, Arch. Rat. Mech. Anal., 101 (1988), 209-260. 